\title{
Parametric inference on step-stress accelerated life testing for the extension of exponential distribution under progressive type-Il censoring
}

\author{
M. M. Mohie El-Din ${ }^{a}$, S. E. Abu-Youssef ${ }^{a}$, Nahed S. A. Ali ${ }^{b}$, A. M. Abd El-Raheem ${ }^{1, b}$ \\ ${ }^{a}$ Department of Mathematics, Faculty of Science, Al-Azhar University, Egypt; \\ ${ }^{b}$ Department of Mathematics, Faculty of Education, Ain Shams University, Egypt
}

\begin{abstract}
In this paper, a simple step-stress accelerated life test (ALT) under progressive type-II censoring is considered. Progressive type-II censoring and accelerated life testing are provided to decrease the lifetime of testing and lower test expenses. The cumulative exposure model is assumed when the lifetime of test units follows an extension of the exponential distribution. Maximum likelihood estimates (MLEs) and Bayes estimates (BEs) of the model parameters are also obtained. In addition, a real dataset is analyzed to illustrate the proposed procedures. Approximate, bootstrap and credible confidence intervals (CIs) of the estimators are then derived. Finally, the accuracy of the MLEs and BEs for the model parameters is investigated through simulation studies.

Keywords: step-stress accelerated life testing, progressive type-Il censoring, Bayes estimation, extension of the exponential distribution, cumulative exposure model, bootstrap confidence interval, simulation study
\end{abstract}

\section{Introduction}

In traditional life testing and reliability experiments, it is not easy to collect lifetimes on highly reliable products with long lifetimes because few or no failures may occur within a limited testing time under normal conditions. Accelerated life test (ALT) is one of the most modern approaches used to obtain adequate failure data in a short period. In such testing, products are tested at higher than usual levels of stress (e.g., temperature, voltage, humidity, vibration or pressure) to induce early failure. The life data collected from such accelerated tests is then analyzed and extrapolated to estimate life characteristics under normal operating conditions. Stress loading in ALT can be applied in different ways, commonly used methods are constant-stress and step-stress. See Nelson (1990) for further information on accelerated models.

In constant-stress ALT, each unit is run at constant high stress until either all units fail or the test terminates. See Nelson (1990) and Mohie El-Din et al. (2016) for more details about constant-stress ALT. In step-stress ALT, the stress on every unit is not fixed but is increased step by step at prespecified times or simultaneous the occurrence of a fixed number of failures. If a test includes two levels of stress referred to as a simple step-stress ALT.

The step-stress models have been studied extensively in the literature. Miller and Nelson (1983) introduced the optimal simple step-stress ALT plans for the exponential distribution in the case of

\footnotetext{
${ }^{1}$ Corresponding author: Department of Mathematics, Faculty of Education, Ain Shams University, Cairo, Egypt.

E-mail: a_m2am@yahoo.com
}

Published 31 July 2016 / journal homepage: http://csam.or.kr

(C) 2016 The Korean Statistical Society, and Korean International Statistical Society. All rights reserved. 
complete data. Bai et al. (1989) studied the optimal simple step-stress ALT plans for the exponential distribution in the case of censoring data. The optimal step-stress test for the exponential distribution with progressive type-I censoring was investigated by Gouno et al. (2004). Balakrishnan et al. (2007) considered the simple step-stress ALT under type-II censoring, assuming a cumulative exposure model for exponential distribution. Srivastava and Shukla (2008a) obtained the optimal plan for simple step-stress ALT under the log-logistic distribution by minimizing the asymptotic variance of the maximum likelihood estimate (MLE) of the median life at the design stress. Srivastava and Shukla (2008b) derived the optimal test plan for simple step-stress under the log-logistic model in the case of censored data. The optimal simple step-stress ALT for truncated logistic distribution with censoring was introduced by Srivastava and Mittal (2010). Ismail (2012) considered the MLEs of parameters of Weibull distribution based on hybrid censored data, assuming a tampered random variable model. Ismail (2014) obtained the MLEs of parameters of Weibull distribution and the acceleration factor under progressive hybrid censoring schemes. Mohie El-Din et al. (2015a) considered the simple stepstress ALT under progressive first-failure censoring, assuming a tampered random variable model for Weibull distribution. Mohie El-Din et al. (2015b) developed Bayes estimation for step-stress ALT to power generalized Weibull distribution under progressive censoring, using a tampered random variable model.

In ALTs, tests are often terminated before all units fail, so that censored data is used to reduce test time and expense. The most two common censoring schemes in life testing or reliability experiments are type-I and type-II censoring. Recently, the progressive type-II censoring scheme has become quite popular for analyzing highly reliable data. This type of censoring scheme can be described as: Suppose $n$ identical items are put to test, the integer $m \leq n$ is a prespecified number of failures and $R_{1}, R_{2}, \ldots, R_{m}$ are $m$ prefixed integers satisfying $R_{1}+R_{2}+\cdots+R_{m}+m=n$. At the time of the first failure $t_{1: m: n}, R_{1}$ of the surviving units are randomly withdrawn. Likewise, at the time of the second failure $t_{2: m: n}, R_{2}$ of the surviving units are randomly withdrawn, and so on. At the time of the $m^{\text {th }}$ failure $t_{m: m: n}$, the experiment is stopped and all surviving $R_{m}=n-m-\left(R_{1}+\cdots+R_{m-1}\right)$ units are withdrawn. For more details about progressive type-II censoring, see Balakrishnan and Aggarwala (2000) and Balakrishnan and Cramer (2014).

The paper is drafted as follows. In Section 2, a description of the lifetime model and test assumptions are presented. In Section 3, the MLEs of model parameters under the simple step-stress ALT are derived. The Bayes estimates (BEs) of model parameters using Markov chain Monte Carlo (MCMC) method are obtained in Section 4. In Section 5, a real dataset is analyzed to illustrate the proposed procedures in Sections 3 and 4. In Section 6, the asymptotic, bootstrap and credible confidence bounds for the model parameters are constructed. Section 7, contains the simulation studies. The conclusion is made in Section 8.

\section{Model description and test assumptions}

\subsection{Extension of the exponential distribution}

In 2011, Nadarajah and Haghighi (2011) introduced an extension of the exponential distribution as an alternative to gamma, Weibull and generalized exponential distributions. It has an increasing as well as a decreasing failure rate depending on the values of the shape parameter. It has an increasing (decreasing) failure rate function when $\gamma>1(\gamma<1)$ respectively, and for $\gamma=1$, it becomes constant.

The extension of the exponential distribution specified by the probability density function (PDF):

$$
f(t)=\gamma \sigma(1+\sigma t)^{\gamma-1} \exp \left\{1-(1+\sigma t)^{\gamma}\right\}, \quad t>0, \gamma>0, \sigma>0,
$$


the corresponding cumulative distribution function $(\mathrm{CDF})$ is

$$
F(t)=1-\exp \left\{1-(1+\sigma t)^{\gamma}\right\}, \quad t>0, \gamma>0, \sigma>0,
$$

and the corresponding hazard rate function (HRF) is given by:

$$
h(t)=\gamma \sigma(1+\sigma t)^{\gamma-1}, \quad t>0, \gamma>0, \sigma>0 .
$$

Then, if $T$ follows the extension of the exponential distribution, we shall denote by $T \sim E E(\gamma, \sigma)$. The exponential distribution is a special case of the $E E$ model when $\gamma=1$. Nadarajah and Haghighi (2011) considered the extension of exponential distribution as a possible alternative to gamma, Weibull and generalized exponential distributions for modeling lifetime data for three reasons. The first reason is based on the relationship between a PDF in (2.1) and its HRF in (2.3). The EE model allows for an increasing HRF when its PDF is monotonically decreasing. In contrast, this property is not valid for gamma, Weibull and generalized exponential distributions. The second reason is the superiority of the $E E$ distribution to model data that has a mode fixed at zero compared to the gamma, Weibull and generalized exponential distributions. The third reason is the $E E$ distribution can be interpreted as a truncated Weibull distribution. If the random variable $Y$ has Weibull distribution with shape parameter $\gamma$ and scale parameter $\sigma$, then the density in equation (2.1) is the same as that of the random variable $Z=Y-\sigma-1$ truncated at zero. The $E E$ distribution can then be interpreted as a truncated Weibull distribution, for more details see Nadarajah and Haghighi (2011).

\subsection{Test assumptions}

Let $S_{0}<S_{1}<S_{2}$ be the stress levels in the test and $S_{0}$ be the use-stress or the design stress. Assume $n$ identical units are tested under stress level $S_{1}$, the surviving units at a prespecified time $\tau$ are put under stress level $S_{2}$. The progressive type-II censoring is applied as: At the time of the first failure $t_{1: m: n}, R_{1}$ units are randomly withdrawn from $n-1$ surviving units. At the time of the second failure $t_{2: m: n}, R_{2}$ units from $n-2-R_{1}$ surviving units are randomly withdrawn. The test is terminated at the time of $m^{\text {th }}$ failure occurs $t_{m: m: n}$, at this time all surviving $R_{m}=n-m-\sum_{j=1}^{m-1} R_{j}$ units are withdrawn. It is clear that the complete samples and type-II censored samples are special cases of this scheme. Let $n_{1}$ be the number of failures before time $\tau$ at stress level $S_{1}$. With these notations the observed progressive censored data is $t_{1: m: n}<t_{2: m: n}<\cdots<t_{n_{1}: m: n}<\tau<t_{n_{1}+1: m: n}<\cdots<t_{m: m: n}$. ALT:

The following assumptions are used throughout the article in the structure of simple step-stress

1. Under stress level $S_{i}, i=0,1,2$, the failure time $T$ follows $E E\left(\gamma, \sigma_{i}\right)$.

2. The relationship between the life characteristic $\sigma$ and the stress loading $S$ takes one of the following forms:

- Arrhenius model: $\ln (\sigma)=a+b /-S, b>0$, where $S$ is the absolute temperature.

- Inverse power model: $\ln (\sigma)=a+b[\ln (S)], b>0$, where $S$ is the voltage.

- Exponential model: $\ln (\sigma)=a+b S, b>0$, where $S$ is a weathering variable.

See Chapter 2 of Nelson (1990) for further information on accelerated models. Thus $\ln (\sigma)$ is a linear function of the transformed stress $\phi(S)=1 /-S, \ln (S)$ or $S$ for the above three models. Furthermore, we assume that the relationship between the parameter $\sigma_{i}$ and the stress level $S_{i}$ is

$$
\ln \left(\sigma_{i}\right)=a+b \phi_{i}, \quad i=0,1,2,
$$


where $a$ and $b(>0)$ are unknown parameters, and $\phi_{i}=\phi\left(S_{i}\right)$ is an increasing function of $S$.

3. The cumulative exposure model holds, see Nelson (1990).

From the assumption of cumulative exposure model and the CDF given in (2.2), the CDF of a test unit under the simple step-stress ALT is

$$
G(t)= \begin{cases}F_{1}(t), & 0<t<\tau, \\ F_{2}\left(t-\tau+\tau^{*}\right), & t \geq \tau,\end{cases}
$$

where $F_{j}(t)=1-\exp \left\{1-\left(1+\sigma_{j} t\right)^{\gamma}\right\}$, for $j=1,2$, and $\tau^{*}$ is the solution of $F_{1}(\tau)=F_{2}\left(\tau^{*}\right)$.

The PDF of (2.5) is given by:

$$
g(t)= \begin{cases}f_{1}(t), & 0<t<\tau \\ f_{2}\left(t-\tau+\tau^{*}\right), & t \geq \tau\end{cases}
$$

where for $j=1,2, f_{j}(t)=\gamma \sigma_{j}\left(1+\sigma_{j} t\right)^{\gamma-1} \exp \left\{1-\left(1+\sigma_{j} t\right)^{\gamma}\right\}$.

\section{Maximum likelihood estimation}

In this section, the MLEs of the model parameters are obtained. Let $t_{i: m: n}=t_{i}$ be the observed values of the lifetime $T$ obtained from a progressive type-II censoring. From the CDF in (2.5) and the corresponding PDF in (2.6), the likelihood function of the three parameters $\gamma, \sigma_{1}$ and $\sigma_{2}$ based on the progressive type-II censoring sample is obtained as:

$$
L\left(\gamma, \sigma_{1}, \sigma_{2}\right)=C\left\{\prod_{i=1}^{n_{1}} f_{1}\left(t_{i}\right)\left[1-F_{1}\left(t_{i}\right)\right]^{R_{i}}\right\}\left\{\prod_{i=n_{1}+1}^{m} f_{2}\left(t_{i}-\tau+\tau^{*}\right)\left[1-F_{2}\left(t_{i}-\tau+\tau^{*}\right)\right]^{R_{i}}\right\},
$$

where $C=n\left(n-1-R_{1}\right)\left(n-2-R_{1}-R_{2}\right) \cdots\left(n-m+1-\sum_{j=1}^{m-1} R_{j}\right)$.

The MLEs of $\gamma, \sigma_{1}$ and $\sigma_{2}$ exist only in case of at least one failure occurs before $\tau$ and after $\tau$, then the logarithm of likelihood function of $\gamma, \sigma_{1}$ and $\sigma_{2}$ is given by:

$$
\begin{aligned}
\ell\left(\gamma, \sigma_{1}, \sigma_{2}\right)= & \log C+n_{1} \log \sigma_{1}+n_{2} \log \sigma_{2}+m \log \gamma+(\gamma-1) \sum_{i=1}^{n_{1}} \log \left(1+\sigma_{1} t_{i}\right) \\
& +\sum_{i=1}^{n_{1}}\left(R_{i}+1\right)\left(1-\left(1+\sigma_{1} t_{i}\right)^{\gamma}\right)+(\gamma-1) \sum_{i=n_{1}+1}^{m} \log \left(1+\sigma_{2} \omega\left(t_{i}\right)\right) \\
& +\sum_{i=n_{1}+1}^{m}\left(R_{i}+1\right)\left(1-\left(1+\sigma_{2} \omega\left(t_{i}\right)\right)^{\gamma}\right),
\end{aligned}
$$

where $\omega\left(t_{i}\right)=t_{i}+\left(\sigma_{1} / \sigma_{2}\right) \tau-\tau$

The likelihood equations of $\gamma, \sigma_{1}$ and $\sigma_{2}$ are respectively

$$
\begin{aligned}
\frac{\partial \ell\left(\gamma, \sigma_{1}, \sigma_{2}\right)}{\partial \gamma}= & \frac{m}{\gamma}+\sum_{i=1}^{n_{1}} \log \left(1+\sigma_{1} t_{i}\right)-\sum_{i=1}^{n_{1}}\left(R_{i}+1\right)\left(1+\sigma_{1} t_{i}\right)^{\gamma} \log \left(1+\sigma_{1} t_{i}\right) \\
& +\sum_{i=n_{1}+1}^{m} \log \left(1+\sigma_{2} \omega\left(t_{i}\right)\right)-\sum_{i=n_{1}+1}^{m}\left(R_{i}+1\right)\left(1+\sigma_{2} \omega\left(t_{i}\right)\right)^{\gamma} \log \left(1+\sigma_{2} \omega\left(t_{i}\right)\right)
\end{aligned}
$$




$$
\begin{aligned}
& \frac{\partial \ell\left(\gamma, \sigma_{1}, \sigma_{2}\right)}{\partial \sigma_{1}}=\frac{n_{1}}{\sigma_{1}}+(\gamma-1) \sum_{i=1}^{n_{1}} \frac{t_{i}}{\left(1+\sigma_{1} t_{i}\right)}-\gamma \sum_{i=1}^{n_{1}}\left(R_{i}+1\right) t_{i}\left(1+\sigma_{1} t_{i}\right)^{\gamma-1}, \\
& \frac{\partial \ell\left(\gamma, \sigma_{1}, \sigma_{2}\right)}{\partial \sigma_{2}}=\frac{n_{2}}{\sigma_{2}}+(\gamma-1) \sum_{i=n_{1}+1}^{m} \frac{t_{i}-\tau}{\left(1+\sigma_{2} \omega\left(t_{i}\right)\right)}-\gamma \sum_{i=n_{1}+1}^{m}\left(R_{i}+1\right)\left(t_{i}-\tau\right)\left(1+\sigma_{2} \omega\left(t_{i}\right)\right)^{\gamma-1} .
\end{aligned}
$$

Now, we have a system of three nonlinear equations in three unknowns $\gamma, \sigma_{1}$ and $\sigma_{2}$. It is obvious that a closed form solution is quite difficult to obtain. Consequently, an iterative procedure such as Newton-Raphson can be used to obtain a numerical solution of the above nonlinear system.

\section{Bayes estimation}

In this section, the square error (SE) loss function and linear exponential (LINEX) loss function are considered to obtain BEs of the model parameters $\gamma, \sigma_{1}$ and $\sigma_{2}$ under progressive type-II censoring. Assume that the model parameters $\gamma, \sigma_{1}$ and $\sigma_{2}$ are independent with priors as:

$$
\begin{gathered}
\pi_{1}(\gamma) \propto \gamma^{\mu_{1}-1} e^{\frac{-\gamma}{\lambda_{1}}}, \quad \gamma>0, \mu_{1}, \lambda_{1}>0, \\
\pi_{2}\left(\sigma_{1}\right) \propto \sigma_{1}^{\mu_{2}-1} e^{\frac{-\sigma_{1}}{\lambda_{2}}}, \quad \sigma_{1}>0, \mu_{2}, \lambda_{2}>0,
\end{gathered}
$$

and

$$
\pi_{3}\left(\sigma_{2}\right) \propto \sigma_{2}^{\mu_{3}-1} e^{\frac{-\sigma_{2}}{\lambda_{3}}}, \quad \sigma_{2}>0, \mu_{3}, \lambda_{3}>0
$$

Many authors used the gamma priors for the scale and the shape parameters instead of the exponential priors because the gamma prior is wealthy enough to cover the prior belief of the experimenter. See Nassar and Eissa (2005) and Kim et al. (2011) for Weibull distribution and Singh et al. (2014a) for the extension of the exponential distribution.

In case of non-informative priors, we take $\mu_{i} \rightarrow 0, i=1,2,3$ and $\lambda_{i} \rightarrow \infty, i=1,2,3$. This technique of transforming the informative priors to non-informative priors was considered by Singh et al. (2014b). Thus the joint prior PDF of $\gamma, \sigma_{1}$ and $\sigma_{2}$ is given by:

$$
\pi\left(\gamma, \sigma_{1}, \sigma_{2}\right) \propto \gamma^{\mu_{1}-1} \sigma_{1}^{\mu_{2}-1} \sigma_{2}^{\mu_{3}-1} e^{-\left(\frac{\gamma}{\lambda_{1}}+\frac{\sigma_{1}}{\lambda_{2}}+\frac{\sigma_{2}}{\lambda_{3}}\right)}, \quad \gamma, \sigma_{1}, \sigma_{2}>0 .
$$

The joint posterior density function of the parameters $\gamma, \sigma_{1}$ and $\sigma_{2}$ can be written from (3.1) and (4.1) as:

$$
\begin{aligned}
\pi^{*}\left(\gamma, \sigma_{1}, \sigma_{2}\right) \propto & L\left(\gamma, \sigma_{1}, \sigma_{2}\right) \pi\left(\gamma, \sigma_{1}, \sigma_{2}\right) \\
\propto & \gamma^{m+\mu_{1}-1} \sigma_{1}^{n_{1}+\mu_{2}-1} \sigma_{2}^{m+\mu_{3}-n_{1}-1} e^{-\left(\frac{\gamma}{\lambda_{1}}+\frac{\sigma_{1}}{\lambda_{2}}+\frac{\sigma_{2}}{\lambda_{3}}\right)}\left\{\prod_{i=1}^{n_{1}} \exp \left\{\left(R_{i}+1\right)\left(1-\left(1+\sigma_{1} t_{i}\right)^{\gamma}\right)\right\}\right. \\
& \left.\times\left(1+\sigma_{1} t_{i}\right)^{\gamma-1}\right\} \prod_{i=n_{1}+1}^{m}\left(1+\sigma_{2} \omega\left(t_{i}\right)\right)^{\gamma-1} \exp \left\{\left(R_{i}+1\right)\left(1-\left(1+\sigma_{2} \omega\left(t_{i}\right)\right)^{\gamma}\right)\right\} .
\end{aligned}
$$

Based on balanced SE loss function, the Bayes estimator of the function of parameters $U=U(\Theta)$, $\Theta=\left(\gamma, \sigma_{1}, \sigma_{2}\right)$ is given by Ahmadi et al. (2009)

$$
\hat{U}_{S E}=\Omega \hat{U}_{M L}+(1-\Omega) \int_{\Theta} U \pi^{*}(\Theta) d \Theta,
$$


where $\hat{U}_{M L}$ is the MLE of $U, \pi^{*}(\Theta)$ is given by equation (4.2) and $0 \leq \Omega<1$.

Based on balanced LINEX loss function, the Bayes estimator of $U=U(\Theta)$ is given

$$
\hat{U}_{L I N E X}=-\frac{1}{c} \ln \left[\Omega e^{-c \hat{U}_{M L}}+(1-\Omega) \int_{\Theta} e^{-c U} \pi^{*}(\Theta) d \Theta\right],
$$

where $c \neq 0$ is the shape parameter of the LINEX loss function.

Unfortunately, we cannot calculate the integrals in equations (4.3) and (4.4) explicitly. Therefore, MCMC technique is used to approximate these integrals.

\subsection{Bayesian estimation using MCMC method}

In this subsection, MCMC method is considered to generate samples from the posterior distribution and then compute the BEs of $\gamma, \sigma_{1}$ and $\sigma_{2}$ under simple step-stress ALT using progressive type-II censoring.

From the joint posterior density function in (4.2), the conditional posterior distributions of $\gamma, \sigma_{1}$ and $\sigma_{2}$ are given respectively by:

$$
\begin{aligned}
\pi^{*}\left(\gamma \mid \sigma_{1}, \sigma_{2}\right) \propto & \gamma^{m+\mu_{1}-1} e^{\frac{-\gamma}{h_{1}}}\left\{\prod_{i=1}^{n_{1}}\left(1+\sigma_{1} t_{i}\right)^{\gamma} \exp \left\{\left(R_{i}+1\right)\left(1-\left(1+\sigma_{1} t_{i}\right)^{\gamma}\right)\right\}\right\} \\
& \times \prod_{i=n_{1}+1}^{m}\left(1+\sigma_{2} \omega\left(t_{i}\right)\right)^{\gamma} \exp \left\{\left(R_{i}+1\right)\left(1-\left(1+\sigma_{2} \omega\left(t_{i}\right)\right)^{\gamma}\right)\right\} \\
\pi^{*}\left(\sigma_{1} \mid \gamma, \sigma_{2}\right) \propto & \sigma_{1}^{n_{1}+\mu_{2}-1} e^{-\frac{\sigma_{1}}{\lambda_{2}}}\left\{\prod_{i=1}^{n_{1}}\left(1+\sigma_{1} t_{i}\right)^{\gamma-1} \exp \left\{\left(R_{i}+1\right)\left(1-\left(1+\sigma_{1} t_{i}\right)^{\gamma}\right)\right\}\right\} \\
& \times \prod_{i=n_{1}+1}^{m}\left(1+\sigma_{2} \omega\left(t_{i}\right)\right)^{\gamma-1} \exp \left\{\left(R_{i}+1\right)\left(1-\left(1+\sigma_{2} \omega\left(t_{i}\right)\right)^{\gamma}\right)\right\}, \\
\pi^{*}\left(\sigma_{2} \mid \gamma, \sigma_{1}\right) \propto & \sigma_{2}^{\mu_{3}+m-n_{1}-1} e^{-\frac{\sigma_{2}}{\lambda_{3}}} \prod_{i=n_{1}+1}^{m}\left(1+\sigma_{2} \omega\left(t_{i}\right)\right)^{\gamma-1} \exp \left\{\left(R_{i}+1\right)\left(1-\left(1+\sigma_{2} \omega\left(t_{i}\right)\right)^{\gamma}\right)\right\} .
\end{aligned}
$$

The conditional posterior distributions of $\gamma, \sigma_{1}$ and $\sigma_{2}$ in (4.5), (4.6) and (4.7) cannot be reduced analytically to well known distribution. The Metropolis-Hastings algorithm is used to generate random samples from these distributions, see Upadhyay and Gupta (2010). The following algorithm is proposed to compute Bayes estimators of $U=U\left(\gamma, \sigma_{1}, \sigma_{2}\right)$ under SE and LINEX loss functions.

\section{Algorithm (1)}

1. Start with $\gamma^{(0)}=\hat{\gamma}_{M L E}, \sigma_{1}^{(0)}=\hat{\sigma}_{1 M L E}, \sigma_{2}^{(0)}=\hat{\sigma}_{2 M L E}$.

2. Set $i=1$.

3. Generate $\gamma^{(i)}, \sigma_{1}^{(i)}$ and $\sigma_{2}^{(i)}$ from equations (4.5)-(4.7) respectively.

4. Set $i=i+1$.

5. Repeat steps (3)-(4) $N$ times. 
6. The approximate means of $U$ and $e^{-c U}$ are given respectively by

$$
\begin{aligned}
E(U) & =\frac{1}{N-M} \sum_{i=M+1}^{N} U\left(\gamma^{(i)}, \sigma_{1}^{(i)}, \sigma_{2}^{(i)}\right), \\
E\left(e^{-c U}\right) & =\frac{1}{N-M} \sum_{i=M+1}^{N} \exp \left\{-c U\left(\gamma^{(i)}, \sigma_{1}^{(i)}, \sigma_{2}^{(i)}\right)\right\},
\end{aligned}
$$

where $M$ is the burn-in period. Then, from equations (4.8) and (4.9), the Bayes estimators of $U$ under balanced SE and balanced LINEX loss functions are given respectively by:

$$
\begin{aligned}
\hat{U}_{S E} & =\Omega \hat{U}_{M L}+(1-\Omega) E(U), \\
\hat{U}_{\text {LINEX }} & =-\frac{1}{c} \ln \left[\Omega e^{-c \hat{U}_{M L}}+(1-\Omega) E\left(e^{-c U}\right)\right] .
\end{aligned}
$$

It is clear that the balanced loss functions are more general, which include the MLE and both symmetric and asymmetric BEs as special cases. For examples, from (4.10), with $\Omega=1$, the Bayes estimate under balanced SE loss function reduces to ML estimate, and for $\Omega=0$, it reduces to the Bayes estimate relative to SE loss function (symmetric). The Bayes estimator under balanced LINEX loss function in (4.11) also reduces to ML estimate when $\Omega=1$, and for $\Omega=0$, it reduces to the case of LINEX loss function (asymmetric). In the case of $0<\Omega<1$, the Bayes estimate under balanced loss functions is a mixture of MLE and symmetric or asymmetric BEs.

\section{Application}

In this section, the estimation procedures were developed in Sections 3 and 4 are illustrated with a real data example.

\subsection{Example}

The data in Table 5.4, of Chapter 5 of Zhu (2010) was collected from a step-stress test of light bulbs with a design stress (voltage) of $2 \mathrm{~V}$. In this test, 64 light bulbs were tested under $2.25 \mathrm{~V}$ for 96 hours; voltage was then increased to $2.44 \mathrm{~V}$. Consequently, the stress change time is $\tau=96$ hours. The failure times of the light bulbs are displayed in Table 1, and the unit removed from test before failure is noted by + . In this case, the number of units under stress is $n=64$, and 11 of these units had removed from the test before the failure. Consequently, the number of failures is $m=53$, the number of failure units under $S_{1}=2.25 \mathrm{~V}$ is $n_{1}=34$, and the progressive censoring scheme $R_{i}, i=1,2, \ldots, 53$ be

$$
R_{i}= \begin{cases}0, & i \neq 35 \\ 11, & i=35\end{cases}
$$

Based on engineering experience, inverse power model is expected to be appropriate to describe the acceleration voltage relationship. Thus, the acceleration model can be expressed as

$$
\ln \left(\sigma_{i}\right)=a+b \ln \left(S_{i}\right), \quad b>0, i=0,1,2 .
$$

To verify the validity of the $E E$ distribution with data in Table 1, we use modified KolmogorovSmirnov goodness-of-fit test for progressive type-II censored data. The modified Kolmogorov-Smirnov 
Table 1: The failure times in hours of 64 light bulbs

\begin{tabular}{cccccccc}
\hline \hline No. & Failure time & No. & Failure time & No. & Failure time & No. & Failure time \\
\hline 1 & 12.07 & 17 & 91.22 & 33 & 14.00 & 49 & 94.38 \\
2 & 19.50 & 18 & 102.10 & 34 & 17.95 & 50 & 97.71 \\
3 & 22.10 & 19 & 105.10 & 35 & 24.00 & 51 & 101.53 \\
4 & 23.11 & 20 & 109.20 & 36 & 26.46 & 52 & 105.11 \\
5 & 24.00 & 21 & 114.40 & 37 & 26.58 & 53 & 112.11 \\
6 & 25.10 & 22 & 117.90 & 38 & 28.06 & 54 & 119.58 \\
7 & 26.90 & 23 & 121.90 & 39 & 34.00 & 55 & 120.20 \\
8 & 36.64 & 24 & 122.50 & 40 & 36.13 & 56 & 126.95 \\
9 & 44.10 & 25 & 123.60 & 41 & 40.85 & 57 & 129.25 \\
10 & 46.30 & 26 & 126.50 & 42 & 41.11 & 58 & 136.31 \\
11 & 54.00 & 27 & 130.10 & 43 & 42.63 & 59 & $140+$ \\
12 & 58.09 & 28 & $140+$ & 44 & 52.51 & 60 & $140+$ \\
13 & 64.17 & 29 & $140+$ & 45 & 62.68 & 61 & $140+$ \\
14 & 72.25 & 30 & $140+$ & 46 & 73.13 & 62 & $140+$ \\
15 & 86.90 & 31 & $140+$ & 47 & 83.63 & 63 & $140+$ \\
16 & 90.09 & 32 & $140+$ & 48 & 91.56 & 64 & $140+$ \\
\hline \hline
\end{tabular}

statistic for progressive type-II censored data was suggested by Pakyari and Balakrishnan (2012). They proposed a general purpose goodness-of-fit test by first estimating the unknown parameters of the hypothesized distribution, then transforming the data to normality, and then testing the goodnessof-fit of the transformed data to normality. For further information about the test procedure and how to transform the data to normality (Pakyari and Balakrishnan, 2012).

Let $T_{1: m: n}<T_{2: m: n}<\cdots<T_{m: m: n}$ be a progressively type-II right censored sample with progressive censoring scheme $\left(R_{1}, R_{2}, \ldots, R_{m}\right)$ from a continuous distribution function $F(t, \theta)$, then the modified Kolmogorov-Smirnov statistic for progressive type-II censored data is given by

$$
D_{m: n}=\max \left\{D_{m: n}^{+}, D_{m: n}^{-}\right\},
$$

where

$$
\begin{aligned}
& D_{m: n}^{+}=\max _{i}\left\{v_{i: m: n}-u_{i: m: n}\right\}, \\
& D_{m: n}^{-}=\max _{i}\left\{u_{i: m: n}-v_{i-1: m: n}\right\},
\end{aligned}
$$

where $v_{i: m: n}=E\left(U_{i: m: n}\right)$ is the expected value of the $i^{\text {th }}$ type-II progressively censored order statistic from the uniform $(0,1)$ distribution, given by

$$
v_{i: m: n}=1-\prod_{j=m-i+1}^{m}\left(\frac{j+R_{m-j+1}+\cdots+R_{m}}{j+1+R_{m-j+1}+\cdots+R_{m}}\right),
$$

and $u_{i: m: n}=F\left(t_{i: m: n}, \hat{\theta}\right)$ for $i=1,2, \ldots, m$.

Based on the technique of Pakyari and Balakrishnan (2012) for transforming the data to normality and then testing the goodness-of-fit of the transformed data to normality, the values of test statistic $\left(D_{m: n}\right)$ and the corresponding $p$-values for each stress level $S_{i}, i=1,2$, are calculated and presented in Table 2. It is clear that the $E E$ distributions provide a good fit to the given data due to all $p$-values be greater than 0.05. The MLEs and BEs of the parameters $\gamma, \sigma_{1}$ and $\sigma_{2}$ are introduced in Table 3, where $\hat{\sigma}_{i}=e^{\hat{a}+\hat{b} \ln \left(S_{i}\right)}, i=1,2$. For this dataset, Bayesian analysis is conducted in case of non-informative priors. 
Table 2: The value of test statistic $D_{m: n}$ and the corresponding $p$-values of each stress level for $E E$ distribution

\begin{tabular}{ccc}
\hline \hline Stress (voltage) & $2.25 \mathrm{~V}$ & $2.44 \mathrm{~V}$ \\
\hline$D_{m: n}$ & 0.1641 & 0.6652 \\
$p$-value & 0.30 & 0.94 \\
\hline \hline
\end{tabular}

Table 3: MLEs and BEs under SE (BSE) and LINEX (BLINEX) loss functions of $\gamma, \sigma_{1}$ and $\sigma_{2}$ for the real dataset

\begin{tabular}{cccccc}
\hline \hline \multirow{2}{*}{ MLEs } & \multirow{2}{*}{ BSE } & \multicolumn{3}{c}{ BLINEX } \\
\cline { 3 - 5 } & & & $c=-2$ & $c=.001$ & $c=2$ \\
\hline$\hat{a}_{M L}$ & $\hat{\sigma}_{1 M L}$ & $\hat{\sigma}_{1 B S}$ & $\hat{\sigma}_{1 B L 1}$ & $\hat{\sigma}_{1 B L 2}$ & $\hat{\sigma}_{1 B L 3}$ \\
$\hat{b}_{M L}$ & $\hat{\sigma}_{2 M L}$ & $\hat{\sigma}_{2 B S}$ & $\hat{\sigma}_{2 B L 1}$ & $\hat{\sigma}_{2 B L 2}$ & $\hat{\sigma}_{2 B L 3}$ \\
$\hat{\gamma}_{M L}$ & & $\hat{\gamma}_{B S}$ & $\hat{\gamma}_{B L 1}$ & $\hat{\gamma}_{B L 2}$ & $\hat{\gamma}_{B L 3}$ \\
\hline-17.798 & 0.00152 & 0.00200622 & 0.00200638 & 0.00200622 & 0.00200606 \\
13.950 & 0.00472 & 0.00608 & 0.006081 & 0.00608 & 0.006079 \\
5.0125 & & 3.99850 & 4.2185 & 3.9984 & 3.7982 \\
\hline \hline
\end{tabular}

MLEs = maximum likelihood estimates; BEs = Bayes estimates; SE = square error; LINEX = linear exponential.

From the acceleration model in (5.1), then the MLE of the scale parameter under normal conditions $\hat{\sigma_{0}}=e^{\hat{a}+\hat{b} \ln \left(S_{0}\right)}=0.000297$. By using the MLEs of $\gamma$ and $\sigma_{0}$, then the failure rate under normal conditions is

$$
h(t)=5.0125 \times 0.000297(1+0.000297 t)^{4.0125}, \quad t>0,
$$

the reliability function under normal conditions is

$$
R(t)=\exp \left\{1-(1+0.000297 t)^{5.0125}\right\}, \quad t>0,
$$

the mean time to failure (MTTF) under normal conditions is

$$
\text { MTTF }=\frac{e^{1}}{0.000297 \times 5.0125} \Gamma\left(\frac{1}{5.0125}, 1\right)=438.978 \text { hours, }
$$

where $\Gamma(\cdot, \cdot)$ is incomplete gamma function.

\section{Interval estimation}

In this section, the approximate, bootstrap and credible confidence intervals (CIs) of the parameters $\gamma, \sigma_{1}$ and $\sigma_{2}$ are derived.

\subsection{Approximate confidence intervals}

In this subsection, the approximate confidence bounds of the parameters are obtained based on the asymptotic distributions of the MLEs of the elements of the vector of unknown parameters $\Theta=$ $\left(\gamma, \sigma_{1}, \sigma_{2}\right)$. It is known that the asymptotic distribution of the MLEs of $\Theta$ is given by Miller (1981).

$$
\left((\hat{\gamma}-\gamma),\left(\hat{\sigma_{1}}-\sigma_{1}\right),\left(\hat{\sigma_{2}}-\sigma_{2}\right)\right) \sim \mathbf{N}(0, \Sigma),
$$

where $\Sigma=\left(\sigma_{i j}\right), i, j=1,2,3$ is the variance-covariance matrix of the unknown parameters $\Theta=$ $\left(\gamma, \sigma_{1}, \sigma_{2}\right)$.

The approximate $100(1-\beta) \%$ two sided CIs for $\theta_{i}$ is given by

$$
\left(\hat{\theta}_{i L}, \hat{\theta}_{i U}\right)=\hat{\theta}_{i} \pm Z_{1-\frac{\beta}{2}} \sqrt{\sigma_{i i}}, \quad i=1,2,3,
$$

where $\left(\hat{\theta}_{1} \equiv \hat{\gamma}, \hat{\theta}_{2} \equiv \hat{\sigma}_{1}, \hat{\theta}_{3} \equiv \hat{\sigma}_{2}\right)$ and $Z_{q}$ is the $100 q^{\text {th }}$ percentile of a standard normal distribution. 


\subsection{Bootstrap confidence intervals}

In this subsection, CIs based on the parametric bootstrap method for the unknown parameters $\gamma, \sigma_{1}$ and $\sigma_{2}$ using percentile interval are derived, for more details see Efron and Tibshirani (1993). The following algorithm is implemented to obtain a bootstrap sample.

\section{Algorithm (2)}

1. From the original data, $\mathbf{t} \equiv\left(t_{1}, \ldots, t_{n_{1}}, t_{n_{1}+1}, \ldots, t_{m}\right)$, compute the MLEs of the parameters $\gamma, \sigma_{1}$ and $\sigma_{2}$.

2. Use $\hat{\gamma}_{M L}, \hat{\sigma}_{1 M L}$ and $\hat{\sigma}_{2 M L}$ to generate a bootstrap sample $\mathbf{t}^{*}$ with same $R_{i}, i=1,2, \ldots, m$.

3. As in step (1) based on $\mathbf{t}^{*}$ compute the bootstrap sample estimates $\hat{\gamma}^{*}, \hat{\sigma}_{1}{ }^{*}$ and $\hat{\sigma}_{2}{ }^{*}$ of $\hat{\gamma}_{M L}, \hat{\sigma}_{1 M L}$ and $\hat{\sigma}_{2 M L}$, respectively.

4. Repeat steps (1)-(3), $B$ times and arrange each estimate in ascending order to obtain the bootstrap samples $\left\{\hat{\gamma}^{*[1]}, \hat{\gamma}^{*[2]}, \ldots, \hat{\gamma}^{*[B]}\right\},\left\{\hat{\sigma}_{1}^{*[1]}, \hat{\sigma}_{1}^{*[2]}, \ldots, \hat{\sigma}_{1}{ }^{*[B]}\right\}$ and $\left\{\hat{\sigma}_{2}{ }^{*[1]}, \hat{\sigma}_{2}{ }^{*[2]}, \ldots, \hat{\sigma}_{2}{ }^{*[B]}\right\}$.

Then, the $100(1-\beta) \%$ percentile bootstrap CIs for $\theta_{i}$ is given by

$$
\left(\hat{\theta}_{i L}^{*}, \hat{\theta}_{i U}^{*}\right)=\left(\hat{\theta}_{i}^{*\left[\beta \frac{B}{2}\right]}, \hat{\theta}_{i}^{*\left[\left(1-\frac{\beta}{2}\right) B\right]}\right), \quad i=1,2,3,
$$

where ${\hat{\theta_{1}}}^{*} \equiv \hat{\gamma}^{*}, \hat{\theta}_{2}{ }^{*} \equiv \hat{\sigma}_{1}{ }^{*}$ and ${\hat{\theta_{3}}}^{*} \equiv \hat{\sigma}_{2}{ }^{*}$.

\subsection{Credible confidence intervals}

A $100(1-\beta) \%$ Bayesian credible or posterior interval for a random quantity $\theta$ is the interval that has the posterior probability $(1-\beta)$ that $\theta$ lies in the interval such that

$$
p(L \leq \theta \leq U)=\int_{L}^{U} \pi^{*}(\theta \mid \mathbf{t}) d \theta=1-\beta .
$$

There are several kinds of credible interval, containing a central interval of posterior probability which is the range of values between the $\beta / 2$ and $(1-\beta) / 2$ percentiles.

The following algorithm is performed to obtain credible CIs of $\gamma, \sigma_{1}$ and $\sigma_{2}$.

\section{Algorithm (3)}

1. Do steps (1)-(6) in Algorithm (1).

2. Repeat step (1), $K$ times and arrange each estimate in ascending order as $\left\{\hat{\theta}_{i S E}^{[1]}, \hat{\theta}_{i S E}^{[2]}, \ldots, \hat{\theta}_{i S E}^{[K]}\right\}$, $i=1,2,3$, where $\hat{\theta}_{1 S E} \equiv \hat{\gamma}_{S E}, \hat{\theta}_{2 S E} \equiv \hat{\sigma}_{1 S E}$ and $\hat{\theta}_{3 S E} \equiv \hat{\sigma}_{2 S E}$.

Then, the $100(1-\beta) \%$ credible CIs for $\theta_{i}$ is given by

$$
\left(\hat{\theta}_{i S E}^{\left[\beta \frac{K}{2}\right]}, \hat{\theta}_{i S E}^{\left[\left(1-\frac{\beta}{2}\right) K\right]}\right), \quad i=1,2,3 .
$$




\section{Simulation studies}

In this section, simulation studies are conducted to investigate the performances of the MLEs and BEs (under square error loss function [SEL] and LINEX loss function [LINEXL]) in terms of their mean square errors (MSEs) and relative absolute biases (RABs) for various choices of $n, m, \tau$ and $R_{i}$, $i=1,2, \ldots, m$. Table 4 introduces MSEs and RABs of the MLEs and BEs of the model parameters; in addition, the $95 \%$ approximate, credible and percentile bootstrap CIs are computed. Table 5 includes the lengths and the coverage probabilities of $95 \%$ approximate, credible and percentile bootstrap CIs of the model parameters. We consider the following three progressive censoring schemes (C.S) for the simulation studies.

$$
\text { Scheme 1: } R_{i}= \begin{cases}n-m, & i=1, \\ 0, & \text { other wise }\end{cases}
$$

$$
\begin{gathered}
\text { Scheme 2: } R_{i}= \begin{cases}1, & i=1,2, \ldots, n-m, \\
0, & \text { other wise. }\end{cases} \\
\text { Scheme 3: If } m \text { even, } R_{i}= \begin{cases}n-m, & i=\frac{m}{2}, \\
0, & \text { other wise. }\end{cases} \\
\text { If } m \text { odd, } R_{i}= \begin{cases}n-m, & i=\frac{m+1}{2}, \\
0, & \text { other wise. }\end{cases}
\end{gathered}
$$

The estimation procedure is performed according to the following algorithm.

\section{Algorithm (4)}

1. Specify the values of $n, m, \tau, \Omega$, and $c$.

2. For given values of the prior parameters $\mu_{1}, \mu_{2}, \mu_{3}, \lambda_{1}, \lambda_{2}$ and $\lambda_{3}$ generate $\gamma$ from $\pi_{1}(\gamma), \sigma_{1}$ from $\pi_{2}\left(\sigma_{1}\right)$ and $\sigma_{2}$ from $\pi_{3}\left(\sigma_{2}\right)$.

3. Generate a simple random sample of size $m$ from $\operatorname{Uniform}(0,1)$ distribution, $\left(U_{1}, U_{2}, \ldots, U_{m}\right)$.

4. Determine the values of the censored scheme $R_{i}, i=1,2, \ldots, m$, such that $\sum_{i=1}^{m} R_{i}=n-m$.

5. Set $E_{i}=U_{i}^{1 /\left(i+\sum_{d=m-i+1}^{m} R_{d}\right)}, i=1,2, \ldots, m$.

6. Obtain the progressive type-II censored sample $\left(U_{1: m: n}^{*}, U_{2: m: n}^{*}, \ldots, U_{m: m: n}^{*}\right)$, where $U_{i: m: n}^{*}=1-$ $\prod_{d=m-i+1}^{m} E_{d}, i=1,2, \ldots, m$.

7. Find $n_{1}$, such that $U_{n_{1}: m: n}^{*}<F_{1}(\tau) \leq U_{n_{1}+1: m: n}^{*}$.

8. From steps (3)-(7), the order observations $t_{1: m: n}, t_{2: m: n}, \ldots, t_{n_{1}: m: n}, t_{n_{1}+1: m: n}, \ldots, t_{m: m: n}$, are calculated as follows

$$
t_{i: m: n}= \begin{cases}\frac{1}{\sigma_{1}}\left\{\left(1-\ln \left(1-U_{i: m: n}^{*}\right)\right)^{\frac{1}{\gamma}}-1\right\}, & i=1,2, \ldots, n_{1}, \\ \tau-\frac{\sigma_{1}}{\sigma_{2}} \tau+\frac{1}{\sigma_{2}}\left\{\left(1-\ln \left(1-U_{i: m: n}^{*}\right)\right)^{\frac{1}{\gamma}}-1\right\}, & i=n_{1}+1, \ldots, m\end{cases}
$$


Table 4: MSEs and RABs inside the parentheses for MLEs and BEs

\begin{tabular}{|c|c|c|c|c|c|c|c|c|}
\hline \multirow{2}{*}{$n$} & \multirow{2}{*}{$m$} & \multirow{2}{*}{ C.S } & \multirow{2}{*}{$\theta$} & \multirow{2}{*}{ ML } & \multirow{2}{*}{ SEL } & \multicolumn{3}{|c|}{ LINEXL } \\
\hline & & & & & & $c=-2$ & $c=.001$ & $c=2$ \\
\hline \multirow{18}{*}{15} & \multirow{18}{*}{12} & \multirow{6}{*}{1} & \multirow[b]{2}{*}{$\sigma_{1}$} & 0.03795 & 0.00052 & 0.00051 & 0.00052 & 0.00053 \\
\hline & & & & $(0.63035)$ & $(0.08776)$ & $(0.08712)$ & $(0.08776)$ & $(0.08841)$ \\
\hline & & & \multirow{2}{*}{$\sigma_{2}$} & 1.06586 & 0.00084 & 0.00086 & 0.00084 & 0.00083 \\
\hline & & & & $(0.8472)$ & $(0.04644)$ & $(0.04673)$ & $(0.04644)$ & $(0.04614)$ \\
\hline & & & \multirow[b]{2}{*}{$\gamma$} & 0.3742 & 0.00076 & 0.00081 & 0.00076 & 0.00072 \\
\hline & & & & $(0.77498)$ & $(0.03927)$ & $(0.04044)$ & $(0.03927)$ & $(0.03812)$ \\
\hline & & \multirow{6}{*}{2} & \multirow[b]{2}{*}{$\sigma_{1}$} & 0.05635 & 0.00048 & 0.00048 & 0.00048 & 0.00049 \\
\hline & & & & $(0.69542)$ & $(0.08322)$ & $(0.08389)$ & $(0.08322)$ & $(0.08506)$ \\
\hline & & & \multirow{2}{*}{$\sigma_{2}$} & 1.04923 & 0.00084 & 0.00086 & 0.00084 & 0.00082 \\
\hline & & & & $(0.83841)$ & $(0.03067)$ & $(0.03099)$ & $(0.03067)$ & $(0.03038)$ \\
\hline & & & \multirow[b]{2}{*}{$\gamma$} & 0.33102 & 0.00275 & 0.00296 & 0.00275 & 0.00255 \\
\hline & & & & $(0.71383)$ & $(0.0791)$ & $(0.08251)$ & $(0.0791)$ & $(0.07572)$ \\
\hline & & & & 0.03762 & 0.00035 & 0.00035 & 0.00035 & 0.00036 \\
\hline & & & $\sigma_{1}$ & $(0.64608)$ & $(0.0725)$ & $(0.07183)$ & $(0.0725)$ & $(0.07317)$ \\
\hline & & 3 & & 1.16453 & 0.02409 & 0.02410 & 0.02409 & 0.02407 \\
\hline & & 3 & $\sigma_{2}$ & $(0.93719)$ & $(0.04651)$ & $(0.04679)$ & $(0.04651)$ & $(0.04622)$ \\
\hline & & & & 0.93459 & 0.00268 & 0.00289 & 0.00268 & 0.00248 \\
\hline & & & $\gamma$ & (1.29368) & $(0.08509)$ & $(0.0885)$ & $(0.08509)$ & $(0.08172)$ \\
\hline & & & & 0.03227 & 0.00034 & 0.00033 & 0.00034 & 0.00033 \\
\hline & & & $\sigma_{1}$ & $(0.55285)$ & $(0.07062)$ & $(0.06996)$ & $(0.07062)$ & $(0.07128)$ \\
\hline & & & & 0.88028 & 0.00079 & 0.00081 & 0.00079 & 0.00077 \\
\hline 15 & 15 & & $\sigma_{2}$ & $(0.83158)$ & $(0.03059)$ & $(0.03083)$ & $(0.03059)$ & $(0.03024)$ \\
\hline & & & & 0.29889 & 0.00049 & 0.00055 & 0.00049 & 0.00043 \\
\hline & & & $\gamma$ & $(0.65396)$ & $(0.03284)$ & $(0.03532)$ & $(0.03284)$ & $(0.03284)$ \\
\hline & & & & 0.03762 & 0.0005 & 0.0005 & 0.0005 & 0.00051 \\
\hline & & & $\sigma_{1}$ & $(0.62092)$ & $(0.08647)$ & $(0.08604)$ & $(0.08647)$ & (0.0869) \\
\hline & & & & 0.9524 & 0.00084 & 0.00085 & 0.00084 & 0.00082 \\
\hline 20 & 15 & 1 & $\sigma_{2}$ & $(0.81362)$ & $(0.03052)$ & $(0.03082)$ & $(0.03052)$ & $(0.03022)$ \\
\hline & & & & 0.22144 & 0.00038 & 0.00039 & 0.00038 & 0.00037 \\
\hline & & & $\gamma$ & $(0.57916)$ & $(0.0275)$ & $(0.02837)$ & $(0.0275)$ & $(0.02685)$ \\
\hline & & & & 0.03718 & 0.00041 & 0.00041 & 0.00041 & 0.00042 \\
\hline & & & $\sigma_{1}$ & $(0.60134)$ & $(0.07829)$ & $(0.07762)$ & $(0.07829)$ & $(0.07896)$ \\
\hline & & 2 & & 0.84186 & 0.00080 & 0.00081 & 0.00080 & 0.00078 \\
\hline & & 2 & $\sigma_{2}$ & $(0.77233)$ & (0.0298) & $(0.0301)$ & (0.0298) & $(0.02951)$ \\
\hline & & & & 0.19224 & 0.00130 & 0.00131 & 0.00130 & 0.00129 \\
\hline & & & $\gamma$ & $(0.5275)$ & $(0.0531)$ & $(0.05334)$ & $(0.0531)$ & $(0.05296)$ \\
\hline & & & & 0.03205 & 0.00035 & 0.00035 & 0.00035 & 0.00036 \\
\hline & & & $\sigma_{1}$ & $(0.56379)$ & $(0.07233)$ & $(0.07167)$ & $(0.07233)$ & $(0.0730)$ \\
\hline & & & & 1.069953 & 0.00087 & 0.00089 & 0.00087 & 0.00085 \\
\hline & & 3 & $\sigma_{2}$ & $(0.8825)$ & $(0.03112)$ & $(0.03142)$ & $(0.03112)$ & $(0.03082)$ \\
\hline & & & & 0.41723 & 0.00239 & 0.00259 & 0.00239 & 0.00220 \\
\hline & & & $\gamma$ & $(0.76791)$ & $(0.07746)$ & $(0.08088)$ & $(0.07746)$ & $(0.07408)$ \\
\hline & & & & 0.02091 & 0.00030 & 0.00028 & 0.00030 & 0.00032 \\
\hline & & & $\sigma_{1}$ & $(0.48696)$ & $(0.07104)$ & $(0.07038)$ & $(0.07104)$ & $(0.07170)$ \\
\hline & & & & 0.72913 & 0.00078 & 0.00080 & 0.00078 & 0.00075 \\
\hline 20 & 20 & & $\sigma_{2}$ & $(0.76653)$ & $(0.0296)$ & $(0.0301)$ & $(0.0296)$ & $(0.0284)$ \\
\hline & & & & 0.455 & 0.00227 & 0.00244 & 0.00227 & 0.00211 \\
\hline & & & $\gamma$ & $(0.50905)$ & $(0.07343)$ & $(0.07664)$ & $(0.07343)$ & $(0.07027)$ \\
\hline & & & & 0.02725 & 0.00045 & 0.00045 & 0.00045 & 0.00046 \\
\hline & & & $\sigma_{1}$ & $(0.52535)$ & $(0.08213)$ & $(0.08147)$ & $(0.08213)$ & $(0.08278)$ \\
\hline 30 & & 1 & & 0.80283 & 0.00083 & 0.00084 & 0.00083 & 0.00081 \\
\hline 30 & 22 & 1 & $\sigma_{2}$ & $(0.77195)$ & $(0.03039)$ & $(0.03066)$ & $(0.03039)$ & $(0.03019)$ \\
\hline & & & & 0.10645 & 0.00035 & 0.00032 & 0.00035 & 0.00037 \\
\hline & & & $\gamma$ & $(0.41491)$ & $(0.02741)$ & $(0.02610)$ & $(0.02741)$ & $(0.02648)$ \\
\hline
\end{tabular}




\begin{tabular}{|c|c|c|c|c|c|c|c|c|}
\hline \multicolumn{9}{|c|}{ Continued } \\
\hline \multirow{2}{*}{$n$} & \multirow{2}{*}{$m$} & \multirow{2}{*}{ C.S } & \multirow{2}{*}{$\theta$} & \multirow{2}{*}{ ML } & \multirow{2}{*}{ SEL } & \multicolumn{3}{|c|}{ LINEXL } \\
\hline & & & & & & $c=-2$ & $c=.001$ & $c=2$ \\
\hline & & \multirow{6}{*}{2} & \multirow[b]{2}{*}{$\sigma_{1}$} & 0.03071 & 0.00041 & 0.00041 & 0.00041 & 0.00042 \\
\hline & & & & $(0.5115)$ & $(0.07820)$ & $(0.07754)$ & $(0.0782)$ & $(0.07886)$ \\
\hline & & & \multirow[b]{2}{*}{$\sigma_{2}$} & 0.82308 & 0.00080 & 0.00082 & 0.00080 & 0.00078 \\
\hline & & & & $(0.72409)$ & $(0.02926)$ & $(0.03016)$ & $(0.02926)$ & $(0.02916)$ \\
\hline & & & \multirow[b]{2}{*}{$\gamma$} & 0.09813 & 0.00125 & 0.00122 & 0.00125 & 0.00128 \\
\hline & & & & $(0.38384)$ & $(0.05216)$ & $(0.05161)$ & $(0.05216)$ & $(0.05279)$ \\
\hline & & \multirow{6}{*}{3} & \multirow[b]{2}{*}{$\sigma_{1}$} & 0.03014 & 0.00032 & 0.00031 & 0.00032 & 0.00032 \\
\hline & & & & $(0.55336)$ & $(0.07235)$ & $(0.07169)$ & $(0.07235)$ & $(0.07301)$ \\
\hline & & & \multirow[b]{2}{*}{$\sigma_{2}$} & 0.79754 & 0.00085 & 0.00086 & 0.00085 & 0.00083 \\
\hline & & & & $(0.7698)$ & $(0.03074)$ & $(0.03103)$ & $(0.03074)$ & $(0.03044)$ \\
\hline & & & \multirow[b]{2}{*}{$\gamma$} & 0.41366 & 0.00220 & 0.00238 & 0.00220 & 0.00203 \\
\hline & & & & $(0.72589)$ & $(0.07476)$ & $(0.08083)$ & $(0.07476)$ & $(0.07326)$ \\
\hline \multirow{6}{*}{30} & \multirow{6}{*}{30} & & \multirow[b]{2}{*}{$\sigma_{1}$} & 0.01746 & 0.00030 & 0.00026 & 0.00030 & 0.00031 \\
\hline & & & & $(0.43587)$ & $(0.07176)$ & $(0.07111)$ & $(0.07176)$ & $(0.07242)$ \\
\hline & & & \multirow[b]{2}{*}{$\sigma_{2}$} & 0.4826 & 0.00080 & 0.00081 & 0.00080 & 0.00077 \\
\hline & & & & $(0.61059)$ & $(0.02762)$ & $(0.02952)$ & $(0.02762)$ & $(0.02491)$ \\
\hline & & & \multirow[b]{2}{*}{$\gamma$} & 0.09491 & 0.000209 & 0.000223 & 0.000209 & 0.000196 \\
\hline & & & & $(0.34028)$ & $(0.02775)$ & $(0.03022)$ & $(0.02775)$ & $(0.02533)$ \\
\hline \multirow{18}{*}{45} & & & & 0.01846 & 0.00043 & 0.00042 & 0.00043 & 0.00044 \\
\hline & & & $\sigma_{1}$ & $(0.47129)$ & $(0.07983)$ & $(0.07917)$ & $(0.07983)$ & $(0.08049)$ \\
\hline & 33 & 1 & & 0.27895 & 0.00065 & 0.00066 & 0.00065 & 0.00064 \\
\hline & 33 & 1 & $\sigma_{2}$ & $(0.50049)$ & $(0.02697)$ & $(0.02718)$ & $(0.02697)$ & $(0.02677)$ \\
\hline & & & & 0.04821 & 0.00024 & 0.00022 & 0.00024 & 0.00025 \\
\hline & & & $\gamma$ & $(0.3029)$ & $(0.02416)$ & $(0.02327)$ & $(0.02416)$ & $(0.02504)$ \\
\hline & & & & 0.01251 & 0.00039 & 0.00037 & 0.00039 & 0.00041 \\
\hline & & & $\sigma_{1}$ & $(0.3225)$ & $(0.07758)$ & $(0.07692)$ & $(0.07758)$ & $(0.07824)$ \\
\hline & & & & 0.25778 & 0.00068 & 0.0007 & 0.00068 & 0.00067 \\
\hline & & 2 & $\sigma_{2}$ & $(0.44797)$ & $(0.02763)$ & $(0.02789)$ & $(0.02763)$ & $(0.02738)$ \\
\hline & & & & 0.03699 & 0.00097 & 0.00095 & 0.00097 & 0.00099 \\
\hline & & & $\gamma$ & $(0.3032)$ & $(0.04967)$ & $(0.04898)$ & $(0.04967)$ & $(0.05035)$ \\
\hline & & & & 0.00707 & 0.00027 & 0.00027 & 0.00027 & 0.00028 \\
\hline & & & $\sigma_{1}$ & $(0.27482)$ & $(0.06337)$ & $(0.06282)$ & $(0.06337)$ & $(0.06392)$ \\
\hline & & & & 0.18425 & 0.00065 & 0.00066 & 0.00065 & 0.00064 \\
\hline & & 3 & $\sigma_{2}$ & (0.40417) & $(0.02697)$ & $(0.02717)$ & $(0.02697)$ & $(0.02675)$ \\
\hline & & & & 0.04554 & 0.00031 & 0.00033 & 0.00031 & 0.00030 \\
\hline & & & $\gamma$ & $(0.29551)$ & $(0.02776)$ & $(0.02837)$ & $(0.02776)$ & (0.02714) \\
\hline & & & & 0.00691 & 0.00020 & 0.00019 & 0.00020 & 0.00020 \\
\hline & & & $\sigma_{1}$ & $(0.0662)$ & $(0.0612)$ & $(0.06058)$ & $(0.0612)$ & $(0.06182)$ \\
\hline 15 & 15 & & & 0.13695 & 0.00053 & 0.00057 & 0.00053 & 0.00051 \\
\hline 45 & 45 & & $\sigma_{2}$ & $(0.35176)$ & $(0.02506)$ & $(0.02527)$ & $(0.02506)$ & $(0.02485)$ \\
\hline & & & & 0.03624 & 0.00021 & 0.00023 & 0.00021 & 0.00020 \\
\hline & & & $\gamma$ & $(0.24853)$ & $(0.02396)$ & $(0.02458)$ & $(0.02396)$ & $(0.02341)$ \\
\hline
\end{tabular}

MSEs = mean square errors; RABs = relative absolute biases; MLEs = maximum likelihood estimates; BEs = Bayes estimates; C.S = censoring scheme; $M L=$ maximum likelihood; $S E L=$ square error loss function; $L I N E X L=$ linear exponential loss function.

True values $\left(\gamma=0.5735, \sigma_{1}=0.2585\right.$ and $\left.\sigma_{2}=0.9455\right)$, values of the prior parameters $\left(\mu_{1}=3.28902, \lambda_{1}=0.1743, \mu_{2}=\right.$ $\left.0.6683, \lambda_{2}=0.3868, \mu_{3}=8.9451, \lambda_{3}=0.1057\right)$, time of changing stress $\tau=13$ and $\Omega=0$.

9. Use the progressively censored data to compute the MLEs of the model parameters by solving the nonlinear system (3.3)-(3.5).

10. Compute the BEs of the model parameters under SE and LINEX loss functions, with $N=11000$, $M=1000$, using Algorithm (1). 
Table 5: Lengths and coverage probabilities of 95\% approximate, credible and bootstrap CIs for $\gamma, \sigma_{1}$ and $\sigma_{2}$

\begin{tabular}{|c|c|c|c|c|c|c|c|c|c|}
\hline \multirow{2}{*}{$n$} & \multirow{2}{*}{$m$} & \multirow{2}{*}{ C.S } & \multirow{2}{*}{$\theta$} & \multicolumn{3}{|c|}{ Length } & \multicolumn{3}{|c|}{ Coverage probability } \\
\hline & & & & Approximate CI & Credible CI & Bootstrap CI & Approximate CI & Credible CI & Bootstrap CI \\
\hline \multirow{9}{*}{15} & \multirow{9}{*}{12} & \multirow{3}{*}{1} & $\sigma_{1}$ & 1.281 & 0.051 & 0.548 & 0.760 & 1 & 0.895 \\
\hline & & & $\sigma_{2}$ & 8.638 & 0.065 & 3.306 & 0.820 & 0.820 & 1 \\
\hline & & & $\gamma$ & 4.160 & 0.168 & 1.504 & 0.980 & 1 & 1 \\
\hline & & \multirow{3}{*}{2} & $\sigma_{1}$ & 1.347 & 0.051 & 0.675 & 0.817 & 1 & 0.950 \\
\hline & & & $\sigma_{2}$ & 7.559 & 0.066 & 3.359 & 0.800 & 0.883 & 1 \\
\hline & & & $\gamma$ & 3.881 & 0.166 & 1.518 & 0.958 & 1 & 1 \\
\hline & & & $\sigma_{1}$ & 1.192 & 0.051 & 0.534 & 0.650 & 1 & 0.912 \\
\hline & & 3 & $\sigma_{2}$ & 7.965 & 0.065 & 3.354 & 0.700 & 0.875 & 1 \\
\hline & & & $\gamma$ & 6.031 & 0.182 & 2.009 & 0.975 & 0.988 & 0.887 \\
\hline \multirow{3}{*}{15} & \multirow{3}{*}{15} & & $\sigma_{1}$ & 1.038 & 0.050 & 0.492 & 0.795 & 1 & 0.970 \\
\hline & & & $\sigma_{2}$ & 8.434 & 0.064 & 3.281 & 0.790 & 0.785 & 1 \\
\hline & & & $\gamma$ & 3.098 & 0.156 & 1.886 & 1 & 1 & 0.930 \\
\hline \multirow{10}{*}{20} & \multirow{9}{*}{15} & \multirow{3}{*}{1} & $\sigma_{1}$ & 1.020 & 0.051 & 0.485 & 0.790 & 0.995 & 0.810 \\
\hline & & & $\sigma_{2}$ & 6.769 & 0.065 & 3.012 & 0.795 & 0.895 & 1 \\
\hline & & & $\gamma$ & 3.370 & 0.162 & 1.391 & 0.980 & 1 & 1 \\
\hline & & \multirow{3}{*}{2} & $\sigma_{1}$ & 1.157 & 0.051 & 0.790 & 0.865 & 1 & 0.970 \\
\hline & & & $\sigma_{2}$ & 7.218 & 0.064 & 3.347 & 0.815 & 0.840 & 1 \\
\hline & & & $\gamma$ & 2.948 & 0.159 & 1.340 & 0.940 & 0.990 & 0.985 \\
\hline & & & $\sigma_{1}$ & 1.116 & 0.050 & 0.511 & 0.850 & 0.995 & 0.945 \\
\hline & & 3 & $\sigma_{2}$ & 7.654 & 0.063 & 3.187 & 0.850 & 0.780 & 1 \\
\hline & & & $\gamma$ & 5.485 & 0.179 & 1.765 & 1 & 1 & 0.920 \\
\hline & \multirow{3}{*}{20} & & $\sigma_{1}$ & 0.803 & 0.050 & 0.484 & 0.845 & 1 & 0.975 \\
\hline \multirow[t]{2}{*}{20} & & & $\sigma_{2}$ & 5.935 & 0.055 & 2.966 & 0.810 & 0.885 & 1 \\
\hline & & & $\gamma$ & 3.410 & 0.159 & 1.693 & 1 & 0.985 & 0.940 \\
\hline \multirow{10}{*}{30} & & & $\sigma_{1}$ & 0.874 & 0.050 & 0.358 & 0.850 & 0.875 & 0.588 \\
\hline & 22 & 1 & $\sigma_{2}$ & 7.688 & 0.064 & 2.872 & 0.887 & 0.750 & 0.988 \\
\hline & & & $\gamma$ & 2.073 & 0.152 & 1.271 & 0.962 & 0.988 & 0.975 \\
\hline & & & $\sigma_{1}$ & 1.101 & 0.051 & 0.710 & 0.930 & 0.970 & 0.990 \\
\hline & & 2 & $\sigma_{2}$ & 6.907 & 0.066 & 3.184 & 0.905 & 0.930 & 1 \\
\hline & & & $\gamma$ & 1.610 & 0.149 & 1.104 & 0.905 & 0.920 & 0.970 \\
\hline & & & $\sigma_{1}$ & 0.796 & 0.051 & 0.469 & 0.820 & 0.990 & 0.935 \\
\hline & & 3 & $\sigma_{2}$ & 5.986 & 0.065 & 3.009 & 0.795 & 0.840 & 1 \\
\hline & & & $\gamma$ & 3.401 & 0.171 & 1.672 & 1 & 1 & 0.930 \\
\hline & & & $\sigma_{1}$ & 0.740 & 0.0501 & 0.337 & 0.875 & 1 & 0.950 \\
\hline 30 & 30 & & $\sigma_{2}$ & 4.738 & 0.065 & 2.354 & 0.812 & 0.862 & 0.988 \\
\hline & & & $\gamma$ & 2.302 & 0.137 & 1.006 & 1 & 0.975 & 0.962 \\
\hline & & & $\sigma_{1}$ & 0.571 & 0.039 & 0.183 & 0.750 & 0.550 & 0.275 \\
\hline 45 & 33 & 1 & $\sigma_{2}$ & 4.235 & 0.052 & 1.446 & 0.925 & 0.950 & 1 \\
\hline & & & $\gamma$ & 1.716 & 0.084 & 0.683 & 1 & 1 & 0.975 \\
\hline & & & $\sigma_{1}$ & 0.778 & 0.047 & 0.369 & 0.975 & 0.825 & 0.950 \\
\hline & & 2 & $\sigma_{2}$ & 4.367 & 0.060 & 1.525 & 0.900 & 1 & 1 \\
\hline & & & $\gamma$ & 1.112 & 0.078 & 0.733 & 0.925 & 0.750 & 0.825 \\
\hline & & & $\sigma_{1}$ & 0.656 & 0.045 & 0.299 & 1 & 1 & 0.950 \\
\hline & & 3 & $\sigma_{2}$ & 4.116 & 0.053 & 1.555 & 0.950 & 1 & 1 \\
\hline & & & $\gamma$ & 1.527 & 0.082 & 0.801 & 1 & 1 & 0.900 \\
\hline & & & $\sigma_{1}$ & 0.476 & 0.029 & 0.149 & 1 & 1 & 0.975 \\
\hline 45 & 45 & & $\sigma_{2}$ & 3.234 & 0.052 & 1.430 & 0.950 & 0.825 & 1 \\
\hline & & & $\gamma$ & 0.190 & 0.071 & 0.589 & 1 & 1 & 1 \\
\hline
\end{tabular}

C.S = censoring schemes; $\mathrm{CI}=$ confidence interval.

True values $\left(\gamma=0.5735, \sigma_{1}=0.2585\right.$ and $\left.\sigma_{2}=0.9455\right)$, time of changing stress $\tau=13$ and $\Omega=0$. 
11. Compute the approximate confidence bounds with confidence level $95 \%$ for the three parameters $\gamma, \sigma_{1}$ and $\sigma_{2}$.

12. Compute 95\% bootstrap and credible CIs using Algorithm (2) and Algorithm (3) respectively.

13. Replicate the steps (3)-(12), 1000 times.

14. Compute the average values of the MSEs and RABs associated with the MLEs and BEs of the parameters $\gamma, \sigma_{1}$ and $\sigma_{2}$.

15. Do steps (1)-(14) with different values of $n, m, \tau$, and $R_{i}, i=1,2, \ldots, m$.

\section{Conclusion}

In this paper, we studied a simple step-stress ALT model for the $E E$ distribution under progressive type-II censored data. Based on simulation studies, point estimation of the model parameters has been investigated through maximum likelihood and Bayes methods. Approximate, credible and bootstrap CIs have been established for the model parameters. The calculations have been made based on different sample sizes and three different progressive censoring schemes.

From the results in Tables 4-5, we observed the following:

1. The MSEs and RABs of MLEs and BEs of the considered parameters decrease as the sample size increases, except for few cases. This may be due to fluctuations in data.

2. The BEs of $\sigma_{1}, \sigma_{2}$ and $\gamma$ give more accurate results through the MSEs and RABs than MLEs.

3. The BEs of $\sigma_{1}$ under LINEX loss function $(c=-2)$ have the smallest MSEs and RABs as compared with estimates under SE loss function, LINEX loss function $(c=2)$, and MLEs.

4. The BEs of $\sigma_{2}$ and $\gamma$ under LINEX loss function $(c=2)$ have the smallest MSEs and RABs compared to estimates under SE loss function, LINEX loss function $(c=-2)$, and MLEs.

5. The length of approximate, bootstrap and credible CIs decreases as the sample size increases, except for a few cases. This may be due to fluctuations in data.

6. The credible CIs of $\sigma_{1}, \sigma_{2}$ and $\gamma$ give more accurate results than approximate and bootstrap CIs through the length and the coverage probability of CIs.

7. The coverage probability of credible CIs of $\sigma_{1}, \sigma_{2}$ and $\gamma$ greater than the coverage probability of approximate and bootstrap CIs, except for few cases. This may be due to fluctuation in data.

8. The bootstrap CIs of $\sigma_{1}, \sigma_{2}$ and $\gamma$ give more accurate results than approximate CIs through the length of CIs.

\section{References}

Ahmadi J, Jozani MJ, Marchand E, and Parsian A (2009). Bayes estimation based on $k$-record data from a general class of distributions under balanced type loss functions, Journal of Statistical Planning and Inference, 139, 1180-1189.

Bai DS, Kim MS, and Lee SH (1989). Optimum simple step-stress accelerated life tests with censoring, IEEE Transactions on Reliability, 38, 528-532. 
Balakrishnan N and Aggarwala R (2000). Progressive Censoring: Theory, Methods, and Applications, Birkhäuser, Boston.

Balakrishnan N and Cramer E (2014). The Art of Progressive Censoring: Applications to Reliability and Quality, Birkhäuser, New York.

Balakrishnan N, Kundu D, Ng HKT, and Kannan N (2007). Point and interval estimation for a simple step-stress model with type-II censoring, Journal of Quality Technology, 39, 35-47.

Efron B and Tibshirani RJ (1993). An Introduction to the Bootstrap, Chapman \& Hall, London.

Gouno E, Sen A, and Balakrishnan N (2004). Optimal step-stress test under progressive type-I censoring, IEEE Transactions on Reliability, 53, 388-393.

Ismail AA (2012). Estimating the parameters of Weibull distribution and the acceleration factor from hybrid partially accelerated life test, Applied Mathematical Modelling, 36, 2920-2925.

Ismail AA (2014). Inference for a step-stress partially accelerated life test model with an adaptive type-II progressively hybrid censored data from Weibull distribution, Journal of Computational and Applied Mathematics, 260, 533-542.

Kim C, Jung J, and Chung Y (2011). Bayesian estimation for the generalized Weibull model under type II progressive censoring, Statistical Papers, 52, 53-70.

Miller R (1981). Survival Analysis, Wiley, New York.

Miller R and Nelson W (1983). Optimum simple step-stress plans for accelerated life testing, IEEE Transactions on Reliability, 32, 59-65.

Mohie El-Din MM, Abu-Youssef SE, Ali NSA, and Abd El-Raheem AM (2015a). Estimation in step-stress accelerated life tests for Weibull distribution with progressive first-failure censoring, Journal of Statistics Applications \& Probability, 3, 403-411.

Mohie El-Din MM, Abu-Youssef SE, Ali NSA, and Abd El-Raheem AM (2015b). Estimation in stepstress accelerated life tests for power generalized Weibull distribution with progressive censoring, Advances in Statistics, 2015, 1-13.

Mohie El-Din MM, Abu-Youssef SE, Ali NSA, and Abd El-Raheem AM (2016). Estimation in constant-stress accelerated life tests for extension of the exponential distribution under progressive censoring, Metron, 7, 1-21.

Nadarajah S and Haghighi F (2011). An extension of the exponential distribution, Statistics, 45, $543-558$.

Nassar MM and Eissa FH (2005). Bayesian estimation for the exponentiated Weibull model, Соттиnications in Statistics - Theory and Methods, 33, 2343-2362.

Nelson W (1990). Accelerated Testing: Statistical Models, Test Plans and Data Analysis, Wiley, New York.

Pakyari R and Balakrishnan N (2012). A general purpose approximate goodness-of-fit test for progressively type-II censored data, IEEE Transactions on Reliability, 61, 238-244.

Singh SK, Singh U, Kumar M, and Vishwakarma PK (2014a). Classical and Bayesian inference for an extension of the exponential distribution under progressive type-II censored data with binomial removals, Journal of Statistics Applications \& Probability Letters, 1, 75-86.

Singh SK, Singh U, and Sharma VK (2014b). Bayesian estimation and prediction for the generalized Lindley distribution under asymmetric loss function, Hacettepe Journal of Mathematics and Statistics, 43, 661-678.

Srivastava PW and Shukla R (2008a). A log-logistic step-stress model, IEEE Transactions on Reliability, 57, 431-434.

Srivastava PW and Shukla R (2008b). Optimum log-logistic step-stress model with censoring, International Journal of Quality \& Reliability Management, 25, 968-976. 
Srivastava PW and Mittal N (2010). Optimum step-stress partially accelerated life tests for the truncated logistic distribution with censoring, Applied Mathematical Modelling, 34, 3166-3178.

Upadhyay SK and Gupta A (2010). A Bayes analysis of modified Weibull distribution via Markov chain Monte Carlo simulation, Journal of Statistical Computation and Simulation, 80, 241-254.

Zhu Y (2010). Optimal design and equivalency of accelerated life testing plans (Doctoral dissertation), Rutgers University, New Brunswick, NJ. 